\title{
Risk, reward, and decision-making in a rodent model of cognitive aging
}

\author{
Ryan J. Gilbert ${ }^{1}$, Marci R. Mitchell ${ }^{1}$, Nicholas W. Simon ${ }^{2}$, Cristina Bañuelos ${ }^{1}$, Barry Setlow ${ }^{3}$ and \\ Jennifer L. Bizon ${ }^{1}$ * \\ ' Department of Neuroscience, University of Florida College of Medicine, Gainesville, FL, USA \\ ${ }^{2}$ Department of Neuroscience, University of Pittsburgh, Pittsburgh, PA, USA \\ ${ }^{3}$ Department of Psychiatry, University of Florida College of Medicine, Gainesville, FL, USA
}

\section{Edited by:}

Gregory R. Samanez-Larkin,

Vanderbilt University, USA

Reviewed by:

Stefan Sandberg, University of

Washington, USA

Paul Gilbert, SDSU-UCSD, USA

*Correspondence:

Jennifer L. Bizon, Department of Neuroscience, University of Florida

College of Medicine, McKnight Brain Institute, P.O. Box 100244,

Gainesville, FL 32610-0244, USA.

e-mail: bizonj@ufl.edu
Impaired decision-making in aging can directly impact factors (financial security, health care) that are critical to maintaining quality of life and independence at advanced ages. Naturalistic rodent models mimic human aging in other cognitive domains, and afford the opportunity to parse the effects of age on discrete aspects of decision-making in a manner relatively uncontaminated by experiential factors. Young adult (5-7 months) and aged (23-25 months) male F344 rats were trained on a probability discounting task in which they made discrete-trial choices between a small certain reward (one food pellet) and a large but uncertain reward (two food pellets with varying probabilities of delivery ranging from 100 to $0 \%)$. Young rats chose the large reward when it was associated with a high probability of delivery and shifted to the small but certain reward as probability of the large reward decreased. As a group, aged rats performed comparably to young, but there was significantly greater variance among aged rats. One subgroup of aged rats showed strong preference for the small certain reward. This preference was maintained under conditions in which large reward delivery was also certain, suggesting decreased sensitivity to reward magnitude. In contrast, another subgroup of aged rats showed strong preference for the large reward at low probabilities of delivery. Interestingly, this subgroup also showed elevated preference for probabilistic rewards when reward magnitudes were equalized. Previous findings using this same aged study population described strongly attenuated discounting of delayed rewards with age, together suggesting that a subgroup of aged rats may have deficits associated with accounting for reward costs (i.e., delay or probability). These deficits in cost-accounting were dissociable from the age-related differences in sensitivity to reward magnitude, suggesting that aging influences multiple, distinct mechanisms that can impact cost-benefit decision-making.

Keywords: aged, choice, rats, probability, discounting, reward sensitivity, memory

\section{INTRODUCTION}

Life requires continuous weighing of costs and benefits to make decisions among outcomes which differ with respect to magnitude, probability, and delay to their arrival. Such choices may prove particularly critical at advanced ages when poor decision-making (e.g., with respect to finances or healthcare) could have deleterious consequences for maintenance of independence and overall quality of life. Many of the neural, cognitive, emotional, and social factors that influence decision-making processes are known to change across the lifespan, but how such alterations integrate to impact decision-making remains poorly understood (Mohr et al., 2010; Eppinger et al., 2011). Such questions are becoming increasingly important, however, given that average life expectancy and the cognitive disabilities associated with advanced age continue to rise (AgingStats.gov, 2005).

Risk-taking has been most often evaluated in aged individuals within the context of economic decisions. Conventional wisdom suggests that risk-taking decreases in normal aging (Kumar, 2007), consistent with evidence that aged individuals report less impulsivity and sensation-seeking than their younger cohorts (Roalf et al., 2011). Indeed, such risk-aversion may be an adaptive strategy under some circumstances (e.g., to preserve accumulated wealth toward the end of life), although excessive risk-aversion could be maladaptive in circumstances in which some degree of risktaking provides a greater net gain. Notably, however, other studies show that aged adults can actually be less likely than young to choose low-risk options in some circumstances (Denburg et al., 2005; Henninger et al., 2010). Broadly speaking, whether decisionmaking improves, declines, or remains stable across the lifespan seems to depend on the type of decision-making and the context in which decisions are framed (Mather, 2006; Mata et al., 2011; Mienaltowski, 2011; Strough et al., 2011). Indeed, relationships between some aspects of decision-making and aging may be non-linear, with decision quality increasing up to approximately age 50 and then declining thereafter (Agarwal et al., 2007). 
Within this context, substantial variability in risk-based decision-making has been reported among aged individuals, implicating multiple cognitive and neural mechanisms (Denburg et al., 2005; Brown and Ridderinkhof, 2009). The degree to which an individual is able to accurately anticipate both future rewards and costs will influence choice behavior (Eppinger et al., 2011); however, studies directly investigating rewards and costs in aging have yielded somewhat disparate results. Gilbert and colleagues reported robust deficits in anticipation of a sucrose reward in aged rats (Maasberg et al., 2011), and Frank and Kong (2008) found enhanced learning about negative compared to positive outcomes in an older compared to a younger subgroup of aged adults. In contrast, Samanez-Larkin et al. (2007) reported that aged subjects showed no difference in their behavioral or neural responses to anticipated rewards but significantly attenuated responses to anticipated costs (although in the same study they found no age difference in subjects' ability to learn about positive vs. negative outcomes). Together, these studies indicate that the effects of age on processing of rewards and costs may be largely dissociable, and support a multiple factor causal framework for age-related changes in decision-making (Brown and Ridderinkhof, 2009). Indeed, deficits in mnemonic abilities are also prevalent among aged individuals, and at least one study found that individual differences in memory and information processing speed could account for some aspects of risk-based decision-making in aged individuals (Henninger et al., 2010).

Naturalistic rodent models mimic human aging in a number of cognitive domains, such as memory and aspects of executive function (e.g., cognitive flexibility). As in humans, there are robust individual differences in cognitive performance among aged rats (Gallagher et al., 1993; Barense et al., 2002; Schoenbaum et al., 2006; LaSarge et al., 2007; Bizon et al., 2009). Because the laboratory rearing environment is largely homogeneous, these individual differences in cognitive aging can be largely dissociated from experiential factors in a way that is difficult to achieve in human populations. In previous work, our laboratory used young and aged Fischer 344 (F344) rats to determine how normal aging affects inter-temporal decision-making, and found that aged rats showed strongly attenuated discounting of delayed rewards relative to young (Simon et al., 2010). To our knowledge, however, there are no studies in which animal models have been used to evaluate the effects of age on risk-based decision-making. In the current study, young and aged F344 rats were assessed on a probability discounting task which involved making discrete-trial choices between small certain rewards and large probabilistically delivered rewards (Cardinal and Howes, 2005; Floresco et al., 2008). The same rats were also assessed in the Morris water maze to determine how age-related alterations in decision-making are related to spatial learning and memory.

\section{MATERIALS AND METHODS SUBJECTS}

Young (5-6 months) and aged (22-24 months) male F344 rats were obtained from the National Institute on Aging colony (Taconic Farms, Hudson, NY, USA) and housed in the AAALACaccredited vivarium facility in the Psychology Building at Texas A\&M University in accordance with the rules and regulations of the Texas A\&M University Laboratory Animal Care Committee. The facility was maintained at a consistent $25^{\circ} \mathrm{C}$ with a $12-\mathrm{h}$ light/dark cycle (lights on at 0800 hours) with free access to food and water except as noted below. Rats were tested in five cohorts (each including at least $n=3$ of each age). These cohorts were tested in the probability discounting and associated control conditions, and a subset was also tested in the Morris water maze, either immediately before or immediately after the decision-making tasks. There was some attrition across experiments, particularly in the aged group, such that only a portion of the rats tested in the probability discounting task completed all of the other tasks.

\section{Experiment 1: assessing the effects of age on probability discounting}

Apparatus. Testing in the probability discounting task and control conditions was conducted in eight identical standard rat behavioral test chambers $(30.5 \mathrm{~cm} \times 25.4 \mathrm{~cm} \times 30.5 \mathrm{~cm}$, Coulbourn Instruments, Whitehall, PA, USA) with metal front and back walls, transparent Plexiglas side walls, and a floor composed of steel rods $(0.4 \mathrm{~cm}$ in diameter $)$ spaced $1.1 \mathrm{~cm}$ apart. Each test chamber was housed in a sound attenuating cubicle, and equipped with a recessed food pellet delivery trough fitted with a photobeam to detect head entries and a 1.12-W lamp to illuminate the trough. This trough, into which the 45-mg grain-based food pellet rewards (PJAI, Test Diet, Richmond, IN, USA) were delivered, was located $2 \mathrm{~cm}$ above the floor in the center of the front wall. Two retractable levers were located to the left and right of the food trough, $11 \mathrm{~cm}$ above the floor. Experiments were controlled and data were collected by a computer interfaced with the behavioral test chambers and equipped with Graphic State 3.01 software (Coulbourn Instruments).

Experimental procedures. Prior to the start of behavioral testing, rats ( $n=20$ young and 20 aged) were reduced to $85 \%$ of their free feeding weight over the course of 1 week, and maintained at this weight for the duration of the experiments (except during water maze training). On the day prior to shaping, each rat was given five $45 \mathrm{mg}$ food pellets in its home cage to reduce neophobia to the food reward used in the task. Shaping procedures for the probability discounting task followed those used previously (Cardinal et al., 2000; LaSarge et al., 2007; Simon et al., 2010). Shaping began with a 64-min session of magazine training consisting of 38 deliveries of a single food pellet with an inter-trial interval (ITI) of $100 \pm 40 \mathrm{~s}$. Following magazine training, rats were trained to press a single lever (either the left or right, counterbalanced across groups; the other lever was retracted during this phase of training) to receive a single food pellet. After reaching a criterion of 50 lever presses in $30 \mathrm{~min}$, rats were then trained on the opposite lever under the same criterion. This protocol was followed by further shaping sessions in which both levers were retracted and rats were trained to nose poke into the food trough during simultaneous illumination of the trough and house lights. When a nose poke occurred, a single lever was extended (left or right, pseudorandomly determined, such that each lever was presented once in every two-trial block), and a lever press resulted in immediate delivery of a single food pellet. Immediately following the lever press, the trough light was extinguished and the lever was retracted. Rats were trained to a 
criterion of 30 presses on each lever within $60 \mathrm{~min}$, with an ITI of $40 \pm 10 \mathrm{~s}$.

Test sessions in the probability discounting task were $60 \mathrm{~min}$ long and contained five blocks of 18 trials each. Each $40 \mathrm{~s}$ trial began with a 10-s illumination of the food trough and house lights. A nose poke into the food trough during this time extinguished the food trough light and triggered extension of either a single lever (forced choice trials) or of both levers simultaneously (free choice trials). If rats failed to nose poke within the 10-s time window, the lights were extinguished and the trial was scored as an omission. A press on one lever (either left or right, counterbalanced across age groups) resulted in immediate delivery of one food pellet (the small reward). A press on the other lever resulted in immediate delivery of two food pellets (the large reward) on a probabilistic basis. The probability of large reward delivery in the first block of trials was set at $100 \%$. In subsequent blocks of trials, the probability of large reward delivery decreased to $75,50,25$, and $0 \%$. Each block began with eight forced choice trials in which only a single lever was extended and which were used to establish the probabilities in effect for that block (four for each lever), followed by 10 free choice trials (Cardinal and Howes, 2005; Simon et al., 2009). Once either lever was pressed, the levers were immediately retracted. Food delivery was accompanied by re-illumination of both the food trough and house lights, which were extinguished upon entry to the food trough to collect the food or after $10 \mathrm{~s}$, whichever occurred sooner. Failure to press either lever within $10 \mathrm{~s}$ of their extension resulted in the levers being retracted and lights extinguished, and the trial was scored as an omission. Rats were tested in the probability discounting task until stable performance was observed across a five session block (at least 25 sessions - see Data Analysis for description of stable performance).

\section{Experiment 2: assessing the effects of age on sensitivity to reward probability (equal rewards condition)}

To assess the rats' ability to detect and respond to the different probabilities of reward delivery employed in the probability discounting task, the amount of food associated with each of the levers was equalized (i.e., one food pellet for either choice) while the probabilities of delivery remained the same as in Experiment 1. Rats were tested under these conditions until stable performance was achieved (at least 10 sessions).

\section{Experiment 3: assessing the effects of age on sensitivity to reward magnitude (equal probabilities condition)}

To assess the rats' ability to detect and respond to differences in reward magnitude, the amounts of food associated with each lever were restored to their initial conditions (one food pellet vs. two food pellets) and the probability of large reward delivery was set to $100 \%$ for all five blocks. Rats were tested under these conditions until stable performance was achieved (at least 10 sessions).

Data analysis. For Experiments 1-3, raw data files were exported from Graphic State software and compiled using a custom macro written for Microsoft Excel (Dr. Jonathan Lifshitz, University of Kentucky). Statistical analysis was conducted in SPSS 19.0. Analyses of stable performance in the decision-making tasks were conducted using a two-factor repeated measures ANOVA (trial block X test session) conducted on the last five consecutive sessions of testing in each experiment. Stable performance was defined as a main effect of trial block in the absence of main effects or interactions involving test session (Mar and Robbins, 2007; Simon et al., 2010). Comparisons between groups in the decision-making tasks were conducted on averaged data collapsed across these last five (stable) sessions, using two-factor repeated measures ANOVA (group $\mathrm{X}$ trial block), with Tukey's post hoc tests when warranted. For all analyses, $p$ values less than 0.05 were considered significant.

\section{Experiment 4: are age-related alterations in decision-making related to spatial memory impairment?}

Apparatus. The water maze consisted of a circular tank (diameter $183 \mathrm{~cm}$, wall height $58 \mathrm{~cm}$ ) painted white and filled with water $\left(27^{\circ} \mathrm{C}\right)$ made opaque with the addition of non-toxic white tempera paint. The maze was surrounded by black curtains to which were affixed large white geometric designs, which provided extramaze visual cues. For the spatial reference memory (hidden platform) task, a retractable escape platform $(12 \mathrm{~cm}$ diameter, HVS Image, UK) was submerged $2 \mathrm{~cm}$ below the surface of the water in the center of the southwest quadrant of the maze. For the cued (visible platform) task, the platform protruded $2 \mathrm{~cm}$ above the surface of the water, and was located in a different quadrant of the maze on each trial. A video camera mounted above the center of the maze was connected to a DVD recorder and computer, which were used for data storage and analysis using a video tracking system (Water 2020, HVS Image, UK).

\section{Procedures.}

Spatial reference memory (hidden platform) task. Spatial reference memory was assessed as described previously (LaSarge et al., 2007; Bizon et al., 2009). Briefly, rats received three daily training trials with a 30-s ITI over eight consecutive days. On each trial, rats were placed into the water facing the wall of the maze at one of four equally spaced start positions (north, south, east, or west). The start positions were varied in a pseudo-random fashion, such that all rats started from each of the locations approximately the same number of times. Once in the water, rats were allowed to swim until they found the hidden platform or until 90 s elapsed, at which time they were guided to the escape platform by the experimenter. Rats remained on the platform for $30 \mathrm{~s}$ and then were placed in a holding chamber for $30 \mathrm{~s}$ before the next trial. Every sixth trial was a probe trial in which the platform was lowered to the bottom of the maze for the first $30 \mathrm{~s}$ of the trial, after which it was raised to allow the rats to escape.

Cued (visible platform) task. On the day after the last session of spatial reference memory training, rats were given a single session with six trials of cue training. For cue training, rats were trained to escape to a visible platform (painted black and protruding $2 \mathrm{~cm}$ above the water's surface). Both the start position and platform location were varied on each trial, making the extramaze cues explicitly irrelevant to the platform location. On each trial, rats were allowed to search for the platform for a maximum of $90 \mathrm{~s}$ and then were allowed to remain there for $30 \mathrm{~s}$ before a 30 -s ITI.

Behavioral and statistical analyses. For each task, data files were created by the Water 2020 software and were exported to Microsoft 
Excel and SPSS (v. 19.0) for analysis. Training trial data in the spatial reference memory task were averaged into four blocks consisting of the five trials preceding each probe trial, and performance was analyzed using a pathlength measure (pathlength is the total distance traveled from the start position to the platform and is reported in centimeters). To provide an overall measure of spatial learning ability for each rat, a "spatial learning index" was calculated using mean search error from interpolated probe trials, as described in Bizon et al. (2009), Gallagher et al. (1993). To calculate search error, the rat's distance from the platform was sampled 10 times/s and these distances were averaged into $1 \mathrm{~s}$ bins. Mean search error is the sum of these $1 \mathrm{~s}$ bins minus the optimal path from the start location to the platform, divided by the 30-s duration of the probe trials. Mean search error on probe trials is weighted and summed to provide the spatial learning index (Gallagher et al., 1993; Bizon et al., 2009). Comparisons between groups on training trials (in both the hidden and visible platform tasks) were conducted using two-factor ANOVA (group X training trial), with Tukey post hoc tests when warranted. In all cases, $p$ values less than 0.05 were considered significant.

\section{RESULTS}

\section{EXPERIMENT 1: ASSESSING THE EFFECTS OF AGE ON PROBABILITY DISCOUNTING}

Rats ( $n=20$ young and 20 aged) were first tested in the probability discounting task, which involved discrete-trial choices between a small certain reward and a large reward for which the probability of delivery decreased in blocks of trials across the course of each test session. As shown in Figure 1A, all rats decreased their choice of the large reward as the probability of reward delivery decreased across trial blocks, but there were no differences between young and aged rats. This was confirmed by a two-factor repeated measures ANOVA (age X probability), which revealed a main effect of probability $\left[F_{(4,152)}=88.06, p<0.05\right]$, but neither a main effect of age $\left[F_{(1,38)}=0.06\right.$, n.s. $]$ nor an interaction between age and probability $\left[F_{(4,152)}=2.14\right.$, n.s. $]$. Notably, there was significantly greater variance in performance among aged rats relative to young (Levene's test for equality of variances conducted on the mean percent choice of the large reward averaged across all five trial blocks, $F=5.30, p<0.05$; Figure 1B). This greater variance in the aged rats fell on both ends of the distribution relative to young rats, suggesting that differences in individual performance may be mediated by multiple underlying factors. To investigate this further, a median split was performed on data from aged rats, creating "highdiscounting" and "low-discounting" subgroups ( $n=10$ /group, Figure 1C). A two-factor repeated measures ANOVA comparing young, aged high-discounting, and aged low-discounting rats revealed a main effect of probability $\left[F_{(4,148)}=91.29, p<0.05\right]$, a main effect of group $\left[F_{(2,37)}=19.03, p<0.05\right]$, and an interaction between probability and group $\left[F_{(8,148)}=7.01, p<0.05\right]$. Post hoc tests revealed that each of the three groups was significantly different from the others $(p s<0.05)$. This subgrouping was used to further investigate behavioral mechanisms underlying the different patterns of discounting observed in aged rats (see below).

\section{EXPERIMENT 2: ASSESSING THE EFFECTS OF AGE ON SENSITIVITY TO PROBABILITY (EQUAL REWARDS CONDITION)}

Differences in discounting of probabilistic rewards could be due to a number of variables, including sensitivity to probability and reward magnitude. To determine whether age-related alterations in preference for probabilistic reward could mediate the increased variance observed in aged rats, the task contingencies were altered such that responses on both levers earned a single food pellet, but the probabilities of reward delivery were the same as in the probability discounting task $(100,75,50,25,0 \%)$. This task condition tested preference for certain vs. probabilistic
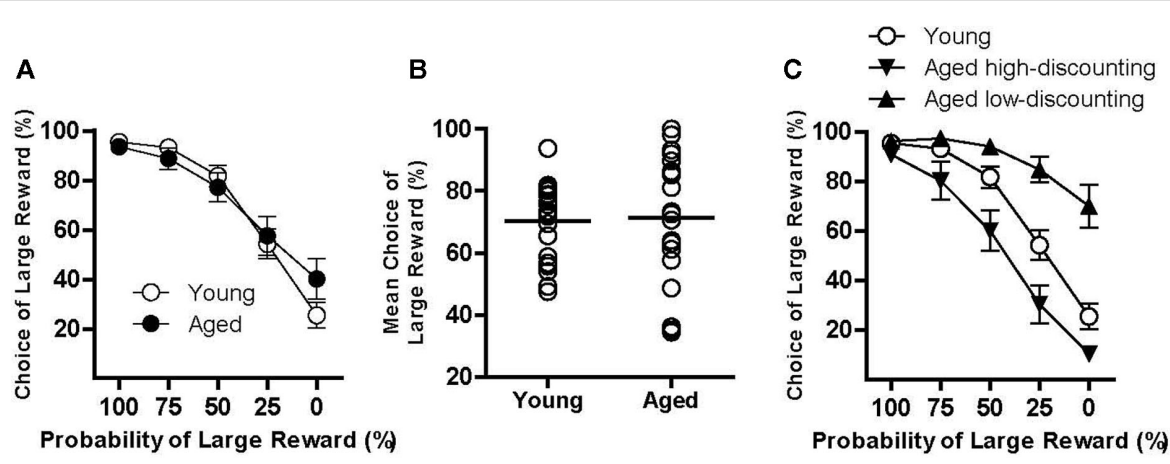

FIGURE 1 | Performance of young and aged rats on the probability discounting task. (A) shows that as a group, both young (open circles) and aged (closed circles) rats discounted the value of the large reward to a comparable degree as indicated by decreased choice of the large reward as the probability of large reward delivery decreased. Notably, however, significantly greater variance in discounting performance was observed among aged rats. (B) shows the mean percent choice of the large reward for individual young and aged rats. By this measure, while some aged rats performed comparably to young, a large subset of aged rats showed a strong preference for the large reward. In contrast, another subset of aged rats showed less preference for the large reward compared to young. Lines indicate median performance in young and aged groups. To confirm that the mean percent choice of the large reward measure reflected true differences in discounting performance, aged rats were subgrouped via a median split into aged high- (downward-facing triangles) and aged low-discounting (upward-facing triangles) subgroups, (C). Note that patterns of responding were significantly different between these two aged subgroups, falling on either side of young performance. This distinction between high- and low-discounting subgroups was used in subsequent conditions to further investigate factors that might contribute to these robustly different patterns of responding. See text for statistical analyses. 
rewards, uncontaminated by differences in reward magnitude. Young $(n=17)$ and aged $(n=15)$ rats were tested in this condition until reaching stable performance. Both young and aged rats decreased their choice of the probabilistic reward as the probability of delivery decreased $\left[F_{(4,120)}=53.90, p<0.05\right]$, but there was no main effect or interaction involving age $(F s<2.30$, n.s.; Figure 2A). There were, however, dramatic differences between the aged high- $(n=7)$ and low- $(n=8)$ discounting rats relative to young rats. Young rats and aged high-discounting rats performed similarly, while aged low-discounting rats showed greater preference for the probabilistic reward than either of the other two groups (Figure 2B). A two-factor ANOVA (group X probability) revealed main effects of both probability $\left[F_{(4,116)}=44.60\right.$, $p<0.05]$ and group $\left[F_{(2,28)}=4.72, p<0.05\right]$, but no interaction $\left[F_{(8,116)}=0.58\right.$, n.s. $]$. Post hoc tests confirmed that the aged lowdiscounting rats had a significantly greater preference for the probabilistic reward compared to the young and aged high-discounting rats $(p s<0.05)$, but that the young and aged high-discounting rats did not differ from each other.

To confirm that the observed differences between aged highand low-discounting rats in the equal rewards condition were not an artifact of the median split of the aged group, the same median split procedure was performed on the young group based on performance in the probability discounting task. As expected, young high- and low-discounting rats differed significantly from each other on the probability discounting task in Experiment 1 [main effect of probability, $F_{(4,72)}=82.99$, $p<0.05$; main effect of group, $F_{(1,18)}=33.26, p<0.05$; interaction between probability and group, $\left.F_{(4,72)}=4.76, p<0.05\right]$. Importantly, however, these groups did not differ in the equal rewards condition [main effect of probability, $F_{(4,60)}=27.81$, $p<0.05$; main effect of group, $F_{(1,15)}=0.05$, n.s.; interaction between probability and group, $F_{(4,60)}=0.88$, n.s.], suggesting that the difference between the aged high- and

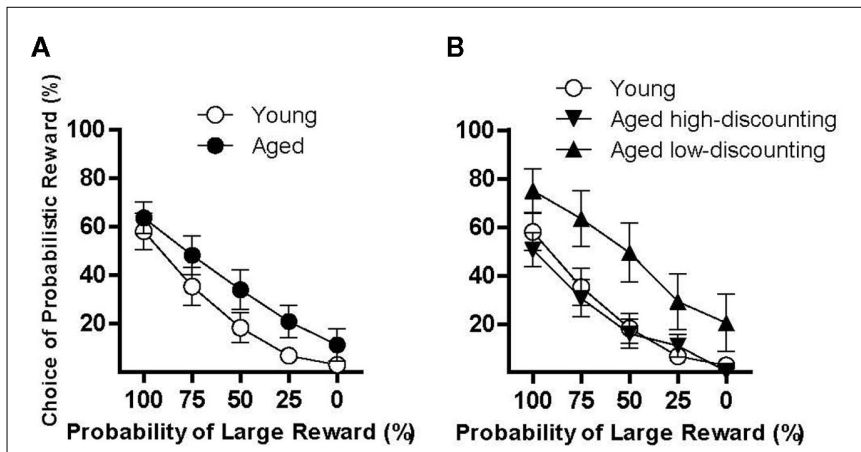

FIGURE 2 | Performance of young and aged rats in the equal rewards condition used to evaluate effects of age on sensitivity to reward probability. (A) shows that in this condition, both young and aged rats discounted the probabilistic reward to a similar degree. However, note that as shown in (B), high- and low-discounting subgroups showed markedly different patterns of responding in this condition. Aged low-discounting rats (upward-facing triangles) maintained a strong preference for the probabilistically delivered reward, whereas both young (open circles) and aged high-discounting (downward-facing triangles) rats strongly discounted this choice. See text for statistical analyses. low-discounting rats on the equal rewards condition was representative of true phenotypic differences between the aged subgroups.

\section{EXPERIMENT 3: ASSESSING THE EFFECTS OF AGE ON SENSITIVITY TO REWARD MAGNITUDE (EOUAL PROBABILITIES CONDITION)}

Data from the equal rewards condition suggested that differential preference for probabilistic rewards could account for some of the variance in probability discounting in the aged rats. To determine whether differential sensitivity to reward magnitude might also contribute to probability discounting performance in aged rats, the task contingencies were altered such that the reward magnitudes were returned to their original condition (one vs. two food pellets), but the probability of the large reward remained at $100 \%$ across all trial blocks. This task condition tested preference for the large vs. small reward, uncontaminated by differences in reward probability. Young $(n=17)$ and aged $(n=11)$ rats were tested in this condition until stable performance was achieved. The pattern of performance differed between young and aged rats, with aged rats showing decreased preference for the large reward across trial blocks relative to young rats twofactor ANOVA (age $\mathrm{X}$ trial block): main effect of trial block $\left[F_{(4,104)}=16.11, p<0.05\right]$, main effect of age $\left[F_{(1,26)}=19.96\right.$, $p<0.05]$, interaction $\left[F_{(8,104)}=3.80, p<0.05\right.$; Figure $\left.\left.3 \mathrm{~A}\right]\right\}$. A similar analysis was also conducted using the aged high- and low-discounting subgroups as in Experiment 2 (Figure 3B). A two-factor ANOVA (group X trial block) revealed a main effect of trial block $\left[F_{(4,100)}=18.99, p<0.05\right]$, as well as a main effect of group $\left[F_{(2,25)}=25.02, p<0.05\right]$ and an interaction between group and trial block $\left[F_{(8,100)}=3.38, p<0.05\right]$. In contrast to the pattern of results in Experiment 2, post hoc tests revealed that aged high-discounting rats $(n=5)$ had significantly reduced preference for the large reward compared to both young rats and aged low-discounting rats $(p s<0.05)$, but that young rats and aged low-discounting $(n=6)$ rats did not differ from each other (n.s.). Importantly, although there was some mortality among aged rats prior to completing Experiments 2 and 3, this mortality likely did not account for differences between aged highand low-discounting subgroups in these experiments, as mortality was equivalent in the two subgroups. Finally, as in the equal rewards condition, there were no differences between the young subgroups in the equal probabilities condition following a median split [main effect of trial block, $F_{(4,60)}=6.81, p<0.05$; main effect of group, $F_{(1,15)}=0.24$, n.s.; interaction between trial block and group, $F_{(4,60)}=1.33$, n.s.].

\section{Relationships between performance on decision-making tasks}

The distinct patterns of differences between the aged high- and low-discounting rats and young rats in the equal rewards and equal probabilities conditions suggest that probability discounting in aged rats was mediated by aged-related alterations in two independent factors. Consistent with this interpretation, among aged rats, both the equal rewards and equal probabilities conditions were correlated with probability discounting task performance (bivariate correlation, $r s=0.61$ and 0.66 respectively, $p s=0.02$ and 0.03$)$, but not with each other $(r=0.40, p=0.22)$. 


\section{EXPERIMENT 4: ARE AGE-RELATED DIFFERENCES IN DECISION-MAKING RELATED TO MEMORY IMPAIRMENT?}

A subset of the rats tested in the choice tasks was also tested in the Morris water maze ( $n=20$ young, $n=17$ aged). Figure $4 \mathrm{~A}$ shows performance (pathlength to reach the hidden platform) on blocks of five training trials in the spatial reference memory task in young and aged rats. A repeated measures ANOVA (age X training trial block) revealed that rats improved over the course of training [main effect of training trial block, $F_{(3,105)}=27.73$, $p<0.05$ ] and that aged rats had significantly longer pathlengths than young cohorts, demonstrating impaired performance [main effect of age, $F_{(1,35)}=14.15, p<0.05$; interaction between age and training trial block, $F_{(3,105)}=7.34, p<0.05$ ].

Performance on the four interpolated probe trials was used to calculate a spatial learning index (Gallagher et al., 1993; Bizon et al., 2009). Learning index scores have been shown to be associated with age-related changes in neurobiological substrates of spatial memory, as well as other aspects of cognition (Nicolle et al., 1999; Smith et al., 2000; Bizon et al., 2001; LaSarge et al., 2007). As expected, an unpaired $t$-test performed on the spatial learning index data indicated that aged rats were significantly impaired (higher learning index scores) relative to young [means $\pm \mathrm{SE}$ : young $=211.0 \pm 7.2$, aged $\left.=267.6 \pm 9.1 ; t_{(35)}=4.91, p<0.05\right]$.

To determine whether impaired water maze performance in the aged rats was specific to spatial learning, rats were trained in a cued (visible platform) version of the water maze task in a single session on the day following the last day of spatial reference memory training. Similar to previous findings in this study population (LaSarge et al., 2007; Bizon et al., 2009; Murchison et al., 2009), there were no differences between young and aged rats in their ability to locate the visible platform [mean $\pm \mathrm{SE}$ pathlength collapsed across the six visible platform training trials: young $=332.3 \pm 23.7$. Aged $=306.5 \pm 37.3 ; t_{(35)}=0.60$, n.s.],

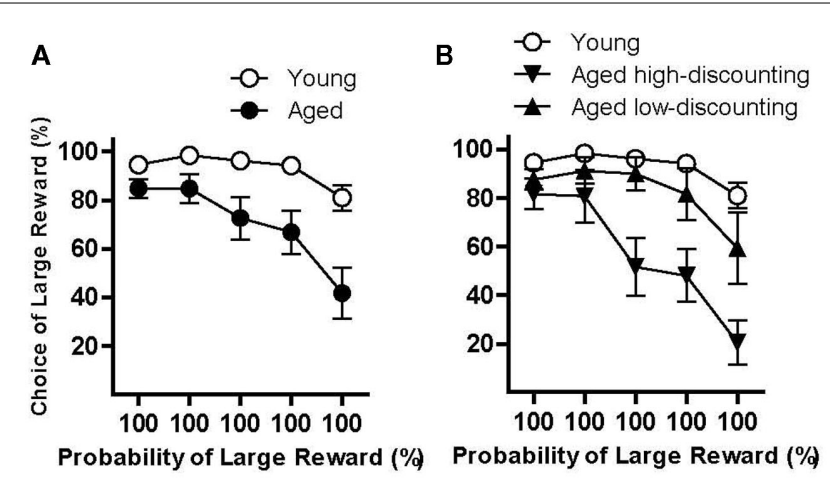

FIGURE 3 | Performance of young and aged rats in the equal probabilities condition used to evaluate effects of age on sensitivity to reward magnitude. (A) shows performance in this condition in which rats were given a choice between one and two food pellets, both with certain delivery. As a group, aged rats showed a decreased preference for the larger reward. Interestingly, as shown in (B), this age difference appeared to be largely mediated by the aged high-discounting rats (downward-facing triangles) as both young (open circles) and aged low-discounting (upward-facing triangles) rats maintained a strong preference for the large reward across trial blocks. See text for statistical analyses. demonstrating that water maze deficits in aged rats were not due to impairments in sensorimotor function, motivation, or ability to learn the procedural aspects of the task.

\section{Relationships between probability discounting and water maze performance}

The results of the analyses described above identified age-related alterations in performance on both the choice tasks and the spatial reference memory version of the Morris water maze. To determine whether performance in these tasks was related (i.e., whether differences in water maze performance could account for individual differences in probability discounting), spatial reference memory performance was compared between aged high- and low-discounting subgroups. A repeated measures ANOVA (subgroup $\mathrm{X}$ training trial block) conducted on training trials revealed main effects of training trial block $\left[F_{(3,102)}=16.81, p<0.05\right]$ and subgroup $\left[F_{(2,34)}=6.91, p<0.05\right]$, as well as a significant interaction $\left[F_{(6,102)}=3.93, p<0.05\right]$. However, post hoc comparisons revealed that these effects were driven by differences between young rats and each of the two aged subgroups $(p s<0.05)$, and that there were no differences between the aged high- and lowdiscounting subgroups with respect to spatial learning ability (Figure 4B). Moreover, a bivariate correlation confirmed that there was no significant relationship among aged rats between mean percent choice of the large reward in the probability discounting task and the spatial learning index in the water maze $(r=0.35$, n.s.).

\section{DISCUSSION}

With the aging of populations in developed countries and the importance of sound decision-making for quality of life, there is increasing interest in understanding and optimizing decisionmaking at advanced ages. Rodent models offer several advantages for addressing such issues, including a relatively short lifespan, the ability to largely control life experience, and the ability to manipulate a range of neurobiological variables. In addition, a large literature indicates that analogous behavioral and neural mechanisms govern animal and human decision-making in young subjects (Floresco et al., 2008; Winstanley, 2011). The experiments

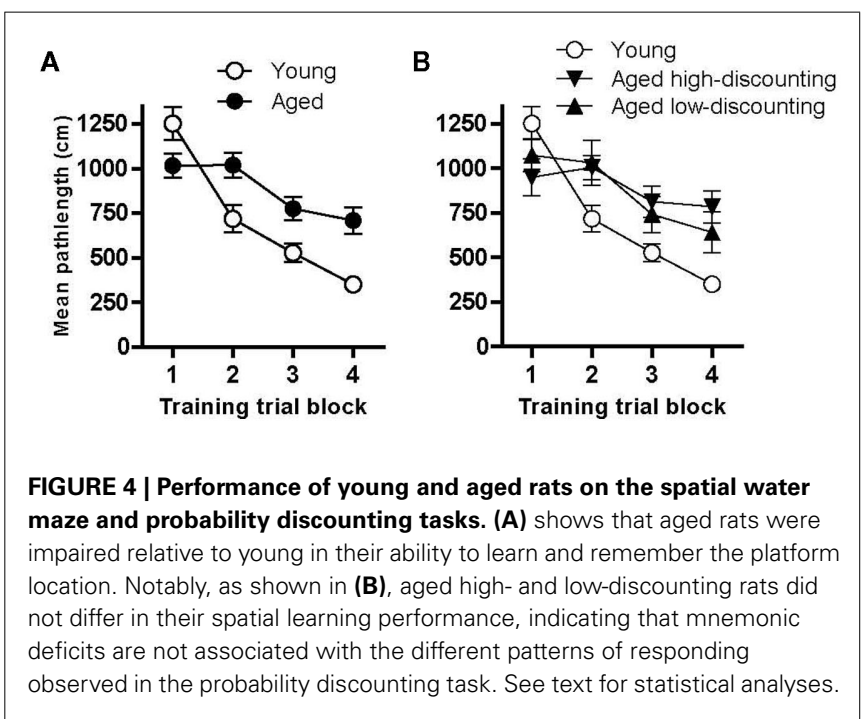


presented here examined the effects of age on a probability discounting task requiring choices between small certain rewards and large rewards for which there were varying risks of reward omission. Although, as a group, aged rats performed comparably to young, there was considerable individual variability in aged rats' performance. Discounting performance in aged (but not young) rats was related to two distinct factors: preference for risky vs. certain rewards (as evident in the equal rewards condition), and preference for large vs. small rewards (as evident in the equal probabilities condition). Importantly, the fact that age-related alterations in these two factors drove performance on the probability discounting task in opposite directions appears to account for the absence of group age differences, and highlights the importance of considering individual differences in studies of cognition in aging (Bizon et al., 2009; Gallagher et al., 2011). Together, these findings suggest that age-related changes in two independent factors (sensitivity to costs and rewards) influence the degree to which probabilistic rewards are discounted (Brown and Ridderinkhof, 2009; Eppinger et al., 2011).

The finding that some aged rats (the low-discounting subgroup) demonstrated elevated preference for probabilistic rewards is consistent with studies in which (some) aged individuals make riskier choices than young, leading to suboptimal outcomes (Denburg et al., 2005; Henninger et al., 2010). This pattern of behavior could reflect impaired perception/discrimination of probabilities in the aged low-discounting subgroup. Another interpretation, however, is that performance in this subgroup reflects a broader deficit in sensitivity to the costs associated with the risky choice, possibly resulting from attenuated negative affect in anticipation of losses (Samanez-Larkin et al., 2007). This latter explanation fits the pattern of results observed previously in our laboratory in which the same study population of F344 rats was assessed on a delay discounting task (Simon et al., 2010). In that study, aged rats discounted delayed rewards to a significantly lesser degree than young rats. Although this pattern of behavior (strong preference for large rewards in spite of the delay to their delivery) was advantageous in the context of the delay discounting task, it could also reflect a failure to account for the costs (having to wait longer for food delivery) incurred by choosing a delayed reward. Indeed, as shown in Figure 5A, a median split performed on the aged rat data from this previous delay discounting study reveals a pattern of results that is similar to that from the present probability discounting experiment (i.e., some aged rats failed to adjust their choice behavior in response to the delay - Figure 5B shows data from Figure 1C replotted for comparison). This similarity is consistent with a subset of aged rats across both experiments failing to account for the costs (delay or probability) associated with the large reward. Future studies in which performance on the delay and probability discounting tasks is compared directly are needed to determine whether deficits in cost-accounting are present in the same subset of aged rats across different types of reward costs.

In addition to possible deficits in cost-accounting, other aged rats (the aged high-discounting subgroup) showed patterns of choice behavior that were consistent with reduced sensitivity to the reward itself. Findings from the human literature regarding reward sensitivity in aging are somewhat contradictory. For example, Samanez-Larkin et al. (2007) reported maintained affective

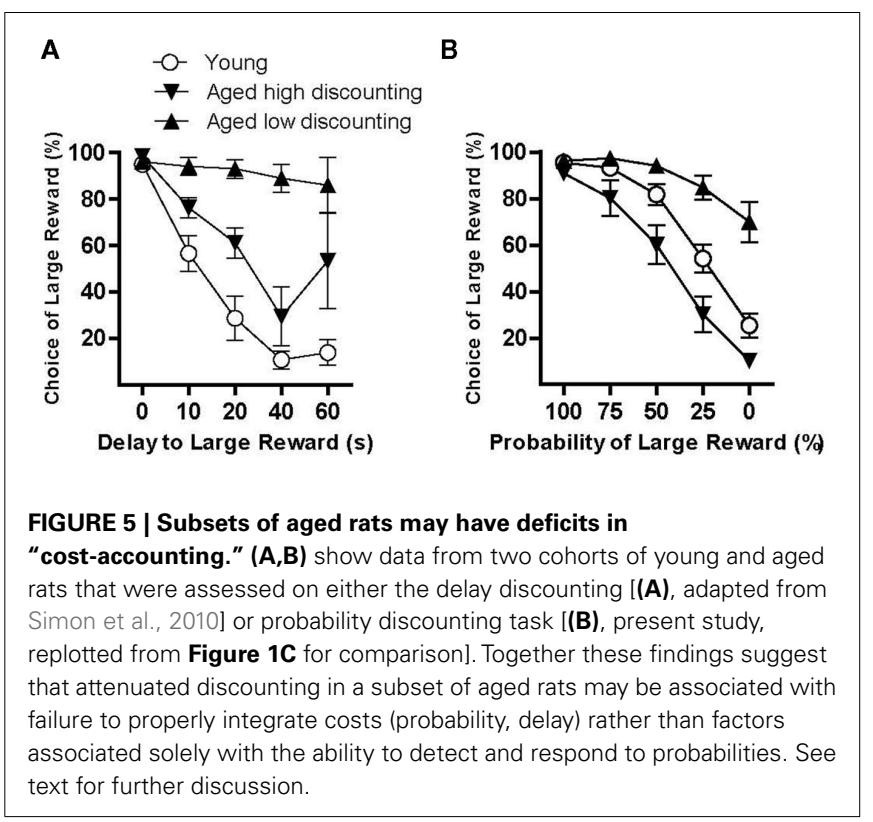

responses in anticipation of gains in aged subjects performing a monetary incentive delay task. In contrast, other studies have reported reduced neural activity and learning in aged subjects' response to rewards, indicating potential reductions in reward sensitivity (Frank and Kong, 2008; Hammerer et al., 2011). Consistent with these latter findings as well as the findings in the present study, a recent paper by Maasberg et al. (2011) found a reduction in reward anticipation in aged rats performing a sucrose preference task. In contrast, in our previous study of delay discounting in aged F344 rats (Simon et al., 2010), we did not find evidence for age-related differences in sensitivity to reward magnitude (aged rats preferred large over small rewards to the same degree as young cohorts). Differences with respect to reward sensitivity in the Simon et al. (2010) study and our current results are likely due to the fact that the magnitude of the difference between the small and large rewards differed across the two studies. In our previous study, the difference between the large (four food pellets) and small (one food pellet) rewards may have been salient enough to overcome any age-related decrements in sensitivity to reward magnitude. In the current study, the smaller difference between the large (two food pellets) and small (one food pellet) rewards may have rendered such sensitivity decrements more readily obvious. Related to this issue, a recent study by Singh et al. (2011) showed no effect of age on a Pavlovian reward devaluation procedure. Devaluation of a food reward via conditioned taste aversion reduced Pavlovian conditioned responding to a cue predictive of that reward to a similar degree in young and aged rats. These findings could be viewed as contrary to age-related changes in representation of reward value; however, in the devaluation paradigm the change in reward value is arguably quite large. Considered together, these data highlight the fact that age-related deficits in reward sensitivity or anticipation are likely to affect behavior to a greater extent when the differences between rewards are small. This hypothesis may help to account for discrepant findings related to age influences on reward sensitivity. 
Probability discounting was correlated with performance in both the equal rewards and equal probabilities conditions, but performance in these two conditions was not related. These findings suggest that sensitivity to costs and sensitivity to reward magnitude are distinct factors that can influence probabilistic decision-making, and that an individual's choice performance may be driven by the relative balance of these two factors (Brown and Ridderinkhof, 2009; Eppinger et al., 2011). Deficits in other cognitive functions in aging also have the potential to influence risk-based decision-making. For example, it has been suggested that due to suboptimal mnemonic abilities, aged subjects tend to rely less on new information and more on previous experience to make decisions (Sanfey and Hastie, 2000; Gilsky, 2007). Indeed, Henninger et al. (2010) showed a relationship between memory and information processing abilities in aged subjects and performance on a risk-based decision task. The current findings did not support such a relationship inasmuch as probability discounting was not correlated with spatial learning in the Morris water maze. Notably, the multiple-day water maze protocol used here is dependent upon hippocampus and related circuitry but is not sensitive to working memory abilities that may be more relevant for probability discounting performance (Sloan et al., 2006). Working memory deficits have been reported in this study population of aged rats, but are not related to individual differences in spatial reference memory (Frick et al., 1995; Bizon et al., 2009). Nevertheless, the fact that performance in the probability discounting and water maze tasks was not correlated indicates that age-related alterations in choice behavior are not secondary to more global cognitive impairments.

In addition to working memory, other cognitive operations mediated by prefrontal cortex, such as cognitive flexibility, decline with age and may impact probability discounting performance (Robbins et al., 1998; Barense et al., 2002; Schoenbaum et al., 2002). Age-related impairments in cognitive flexibility are of particular note because aged rats' performance in the present study could be viewed as "inflexible," in that the degree of preference for the large reward in both aged subgroups tended to remain similar across the three choice conditions. While possible, several lines of evidence argue against this interpretation. First, when the contingencies changed across the three conditions, all young and aged rats shifted their performance to a significant degree (compare Figures 1C, 2B, and 3B). Second, despite the fact that performance in both the equal rewards and equal probabilities conditions was correlated with probability discounting, they were not correlated with each other. This lack of relationship was observed despite the fact that these two conditions occurred in close temporal proximity. Third, in our previous study of delay discounting in young and aged rats, we found no evidence for perseverative behavior across many additional task conditions (Simon et al., 2010). Together, these findings argue against explicitly perseverative behavior as the sole mediator of the current results. It remains possible, however, that elevated preference for the large reward in the aged low-discounting subgroup in the probability discounting task was due in part to some form of impaired cognitive flexibility (specifically a reduced ability to alter choice behavior in response to the within-session changes in reward contingencies). Previous findings reporting that a subset of aged rats show impaired cognitive flexibility in other tasks are consistent with this possibility (Barense et al., 2002; Schoenbaum et al., 2006).

The dopaminergic system has been strongly linked to riskbased decision-making (Brown and Ridderinkhof, 2009; Mohr et al., 2010). Stimulation of both D1 and D2 dopamine receptors increases preference for the large risky reward in the probability discounting task in rats, while stimulation of D3 receptors has the opposite effect (St Onge and Floresco, 2009). In addition, the activity of midbrain dopamine neurons encodes information regarding both reward probability and delay (Fiorillo et al., 2003; Kobayashi and Schultz, 2008), suggesting that this neurochemical system processes information regarding outcome costs. Consistent with this hypothesis, a recent study found that suboptimal increases in preference for the large risky reward in the probability discounting task in a chronic ethanol exposure model were associated with a failure of mesolimbic dopamine activity to encode information about risk of reward omission (Nasrallah et al., 2011). Given that dopaminergic neurotransmission is attenuated with age (Burwell et al., 1995; Kaasinen and Rinne, 2002), it is possible that reductions in dopaminergic encoding of reward costs could account for the increased preference for the risky reward in some rats in the present study. Serotonergic systems have also been linked to decision-making processes. Although serotonin depletion appears to have minimal effects on probability discounting (Mobini et al., 2000; Anderson et al., 2003), it can affect reward sensitivity by reducing discrimination between different reward magnitudes and/or by enhancing the effects of punishment relative to reward (Rogers et al., 2003; Cools et al., 2008). Given that serotonergic signaling appears to decline with age (Arranz et al., 1993; Wang et al., 1995), such deficits could account for the decreased preference for the large reward observed in a subgroup of aged rats in the present study. Importantly, this distinction between the possible functions of the dopaminergic and serotonergic systems is not absolute (e.g., dopaminergic signaling also encodes reward magnitude, and serotonin can modulate dopaminergic activity); nevertheless, it provides a framework for future investigation of the neural mechanisms of age-related alterations in decision-making (Eppinger et al., 2011).

The study presented here is, to our knowledge, the first to investigate the effects of aging on risk-based decision-making in an animal model. The results indicate substantial variability in preference for large risky vs. small guaranteed rewards in aged rats, which appears to be mediated by two distinct factors (sensitivity to costs, and sensitivity to reward). While deficits in both of these factors were observed among the aged cohort, they appeared largely dissociable, and neither was evident in all subjects. These findings suggest that variations in (at least) these two factors may account for altered decision-making at advanced ages, consistent with evidence from studies in humans (Brown and Ridderinkhof, 2009). In addition, the findings of robust individual differences in probability discounting in aged rats are consistent with evidence for individual differences in aged rat performance in other cognitive domains (Gallagher et al., 1993, 2011; Barense et al., 2002; Schoenbaum et al., 2006; Bizon et al., 2009), as well as with evidence for both impaired and preserved 
decision-making abilities in subsets of aged humans (Denburg et al., 2005, 2007). Such findings highlight the importance of taking into account individual differences when investigating cognitive aging.

\section{REFERENCES}

Agarwal, S., Driscoll, J. C., Gabaix, X., and Laibson, D. (2007). The Age of Reason: Financial Decision Over the Life Cycle. Cambridge: Department of Ecomonics, Massachusetts Institute of Technology.

AgingStats.gov. (2005). Older Americans 2004: Key Indicators of WellBeing. Available at: http://www.agi ngstats.gov/agingstatsdotnet/Main_ Site/Data/Data_2004.aspx

Anderson, I. M., Richell, R. A., and Bradshaw, C. M. (2003). The effect of acute tryptophan depletion on probabilistic choice. J. Psychopharmacol. (Oxford) 17, 3-7.

Arranz, B., Eriksson, A., Mellerup, E., Plenge, P., and Marcusson, J. (1993). Effect of aging in human cortical preand postsynaptic serotonin binding sites. Brain Res. 620, 163-166.

Barense, M. D., Fox, M. T., and Baxter, M. G. (2002). Aged rats are impaired on an attentional set-shifting task sensitive to medial frontal cortex damage in young rats. Learn. Mem. 9, 191-201.

Bizon, J. L., Helm, K. A., Han, J. S., Chun, H. J., Pucilowska, J., Lund, P. K., and Gallagher, M. (2001). Hypothalamic-pituitaryadrenal axis function and corticosterone receptor expression in behaviourally characterized young and aged Long-Evans rats. Eur. J. Neurosci. 14, 1739-1751.

Bizon, J. L., LaSarge, C. L., Montgomery, K. S., McDermott, A. N., Setlow, B., and Griffith, W. H. (2009). Spatial reference and working memory across the lifespan of male Fischer 344 rats. Neurobiol. Aging 30, 646-655.

Brown, S. B., and Ridderinkhof, $\mathrm{K}$. R. (2009). Aging and the neuroeconomics of decision making: a review. Cogn. Affect. Behav. Neurosci. 9, 365-379.

Burwell, R. D., Lawler, C. P., and Gallagher, M. (1995). Mesostriatal dopamine markers in aged Long-Evans rats with sensorimotor impairment. Neurobiol. Aging 16, 175-186.

Cardinal, R. N., and Howes, N. J. (2005). Effects of lesions of the nucleus accumbens core on choice between small certain rewards and large uncertain rewards in rats. BMC Neurosci. 6, 37. doi:10.1186/1471-2202-6-37
Cardinal, R. N., Robbins, T. W. and Everitt, B. J. (2000). The effects of d-amphetamine, chlordiazepoxide, alpha-flupenthixol and behavioural manipulations on choice of signalled and unsignalled delayed reinforcement in rats. Psychopharmacology (Berl.) 152, 362-375.

Cools, R., Roberts, A. C., and Robbins, T. W. (2008). Serotoninergic regulation of emotional and behavioural control processes. Trends Cogn. Sci. (Regul. Ed.) 12, 31-40.

Denburg, N. L., Cole, C. A., Hernandez, M., Yamada, T. H., Tranel, D., Bechara, A., and Wallace, R. B. (2007). The orbitofrontal cortex, real-world decision-making, and normal aging. Ann. N. Y. Acad. Sci. 1121, 480-498.

Denburg, N. L., Tranel, D., and Bechara, A. (2005). The ability to decide advantageously declines prematurely in some normal older persons. Neuropsychologia 43, 1099-1106.

Eppinger, B., Hammerer, D., and Li, S. C. (2011). Neuromodulation of reward-based learning and decision making in human aging. Ann. N. Y. Acad. Sci. 1235, 1-17.

Fiorillo, C. D., Tobler, P. N., and Schultz, W. (2003). Discrete coding of reward probability and uncer299, 1898-1902.

Floresco, S. B., St Onge, J. R., GhodsSharifi, S., and Winstanley, C. A. (2008). Cortico-limbic-striatal circuits subserving different forms of cost-benefit decision making. $\operatorname{Cog} n$. Affect. Behav. Neurosci. 8, 375-389.

Frank, M. J., and Kong, L. (2008). Learning to avoid in older age. Psychol. Aging 23, 392-398.

Frick, K. M., Baxter, M. G., Markowska, A. L., Olton, D. S., and Price, D. L. (1995). Age-related spatial reference and working memory deficits assessed in the water maze. Neurobiol. Aging 16, 149-160.

Gallagher, M., Burwell, R., and Burchinal, M. (1993). Severity of spatial learning impairment in aging: development of a learning index for performance in the Morris water maze. Behav. Neurosci. 107, 618-626.

Gallagher, M., Stocker, A. M., and Koh, M. T. (2011). Mindspan: lessons from rat models of neurocognitive aging. ILAR J. 52, 32-40. tainty by dopamine neurons. Science

\section{ACKNOWLEDGMENTS}

Supported by AG029421 (Jennifer L. Bizon and Barry Setlow), DA023331 (Nicholas W. Simon), and the McKnight Brain Research Foundation (Jennifer L. Bizon).

Gilsky, E. L. (2007). "Changes in cognitive function in human aging," in Brain Aging: Models, Methods, and Mechanisms, p. Chapter 1, ed. D. R. Riddle (Boca Raton: CRC Press), 3-20.

Hammerer, D., Li, S. C., Muller, V., and Lindenberger, U. (2011). Life span differences in electrophysiological correlates of monitoring gains and losses during probabilistic reinforcement learning. J. Cogn. Neurosci. 23, 579-592.

Henninger, D. E., Madden, D. J., and Huettel, S. A. (2010). Processing speed and memory mediate agerelated differences in decision making. Psychol. Aging 25, 262-270.

Kaasinen, V., and Rinne, J. O. (2002). Functional imaging studies of dopamine system and cognition in normal aging and Parkinson's disease. Neurosci. Biobehav. Rev. 26, 785-793.

Kobayashi, S., and Schultz, W. (2008). Influence of reward delays on responses of dopamine neurons. $J$. Neurosci. 28, 7837-7846.

Kumar, A. (2007). Who gambles in the stock market? Austin: McCombs School of Business, University of Texas at Austin.

LaSarge, C. L., Montgomery, K. S., Tucker, C., Slaton, G. S., Griffith, W. H., Setlow, B., and Bizon, J. L. (2007). Deficits across multiple cognitive domains in a subset of aged Fischer 344 rats. Neurobiol. Aging 28, 928-936.

Maasberg, D. W., Shelley, L. E., Gracian, E. I., and Gilbert, P. E. (2011). Agerelated differences in the anticipation of future rewards. Behav. Brain Res. 223, 371-375.

Mar, A. C., and Robbins, T. W. (2007). Delay discounting and impulsive choice in the rat. Curr. Protoc. Neurosci. Chapter 8, Unit 8.22.

Mata, R., Josef, A. K., Samanez-Larkin, G. R., and Hertwig, R. (2011). Age differences in risky choice: a metaanalysis. Ann. N. Y. Acad. Sci. 1235, 18-29.

Mather, M. (2006). "A review of decision-making processes: weighing the risks and benefits of aging," in When I'm 64, eds L. L. Carstensen and C. R. Hartel (Washington, DC: The National Academies Press), 145-173.

Mienaltowski, A. (2011). Everyday problem solving across the adult life span: solution diversity and efficacy. Ann. N. Y. Acad. Sci. 1235, 75-85.

Mobini, S., Chiang, T. J., Ho, M. Y., Bradshaw, C. M., and Szabadi, E. (2000). Effects of central 5hydroxytryptamine depletion on sensitivity to delayed and probabilistic reinforcement. Psychopharmacology (Berl.) 152, 390-397.

Mohr, P. N., Li, S. C., and Heekeren, H. R. (2010). Neuroeconomics and aging: neuromodulation of economic decision making in old age. Neurosci. Biobehav. Rev. 34, 678-688.

Murchison, D., McDermott, A. N., Lasarge, C. L., Peebles, K. A., Bizon, J. L., and Griffith, W. H. (2009). Enhanced calcium buffering in F344 rat cholinergic basal forebrain neurons is associated with age-related cognitive impairment. J. Neurophysiol. 102, 2194-2207.

Nasrallah, N. A., Clark, J. J., Collins, A. L., Akers, C. A., Phillips, P. E., and erence following adolescent alcohol use is associated with corrupted encoding of costs but not rewards by mesolimbic dopamine. Proc. Natl. Acad. Sci. U.S.A. 108, 5466-5471.

Nicolle, M. M., Colombo, P. J., Gallagher, M., and McKinney, M. (1999). Metabotropic glutamate receptor-mediated hippocampal phosphoinositide turnover is aged rats. J. Neurosci. 19, 9604-9610.

Roalf, D. R., Mitchell, S. H., Harbaugh, W. T., and Janowsky, J. S. (2011). Risk, reward, and economic decision making in aging. $J$. Gerontol. B. Psychol. Sci. Soc. Sci. doi: 10.1093/geronb/gbr099. [Epub ahead of print].

Robbins, T. W., James, M., Owen, A. M., Sahakian, B. J., Lawrence, A. D., McInnes, L., and Rabbitt, P. M (1998). A study of performance on tests from the CANTAB battery sensitive to frontal lobe dysfunction in a large sample of normal volunexecutive functioning and cognitive aging. Cambridge Neuropsychological Test Automated Battery. J. Int. Neuropsychol. Soc. 4, 474-490.

Rogers, R. D., Tunbridge, E. M., Bhagwagar, Z., Drevets, W. C., Sahakian, B. J., and Carter, C. S. (2003). Tryptophan depletion alters the decision-making of healthy volunteers through altered processing of Bernstein, I. L. (2011). Risk prefblunted in spatial learning-impaired teers: implications for theories of 
reward cues. Neuropsychopharmacology 28, 153-162.

Samanez-Larkin, G. R., Gibbs, S. E., Khanna, K., Nielsen, L., Carstensen, L. L., and Knutson, B. (2007). Anticipation of monetary gain but not loss in healthy older adults. Nat. Neurosci. 10, 787-791.

Sanfey, A. G., and Hastie, R. (2000). "Judgment and decision making across the adult life span: a tutorial review of psychological research," in Cognitive Aging: A Primer, eds D. Park and N. Schwarz (Philadelphia: Psychology Press), 253.

Schoenbaum, G., Nugent, S., Saddoris, M. P., and Gallagher, M. (2002). Teaching old rats new tricks: agerelated impairments in olfactory reversal learning. Neurobiol. Aging 23, 555-564.

Schoenbaum, G., Setlow, B., Saddoris, M. P., and Gallagher, M. (2006). Encoding changes in orbitofrontal cortex in reversal-impaired aged rats. J. Neurophysiol. 95, 1509-1517.

Simon, N. W., Gilbert, R. J., Mayse, J. D., Bizon, J. L., and Setlow, B.
(2009). Balancing risk and reward: a rat model of risky decision making. Neuropsychopharmacology 34, 2208-2217.

Simon, N. W., LaSarge, C. L., Montgomery, K. S., Williams, M. T., Mendez, I. A., Setlow, B., and Bizon, J. L. (2010). Good things come to those who wait: attenuated discounting of delayed rewards in aged Fischer 344 rats. Neurobiol. Aging 31, 853-862.

Singh, T., Jones, J. L., McDannald, M. A., Haney, R. Z., Cerri, D. H., and Schoenbaum, G. (2011). Normal aging does not impair orbitofrontaldependent reinforcer devaluation effects. Front. Aging Neurosci. 3:4. doi:10.3389/fnagi.2011.00004

Sloan, H. L., Good, M., and Dunnett, S. B. (2006). Double dissociation between hippocampal and prefrontal lesions on an operant delayed matching task and a water maze reference memory task. Behav. Brain Res. 171, 116-126.

Smith, T. D., Adams, M. M., Gallagher, M., Morrison, J. H., and Rapp, P. R. (2000). Circuit- specific alterations in hippocampal synaptophysin immunoreactivity predict spatial learning impairment in aged rats. J. Neurosci. 20, 6587-6593.

St Onge, J. R., and Floresco, S. B. (2009). Dopaminergic modulation of risk-based decision making. Neuropsychopharmacology 34, 681-697.

Strough, J., Karns, T. E., and Schlosnagle, L. (2011). Decision-making heuristics and biases across the life span. Ann. N. Y. Acad. Sci. 1235 , 57-74.

Wang, G. J., Volkow, N. D., Logan, J., Fowler, J. S., Schlyer, D., MacGregor, R. R., Hitzemann, R. J., Gur, R. C., and Wolf, A. P. (1995). Evaluation of age-related changes in serotonin 5-HT2 and dopamine D2 receptor availability in healthy human subjects. Life Sci. 56:PL249-53.

Winstanley, C. A. (2011). The utility of rat models of impulsivity in developing pharmacotherapies for impulse control disorders. Br. J. Pharmacol. 164, 1301-1321.
Conflict of Interest Statement: The authors declare that the research was conducted in the absence of any commercial or financial relationships that could be construed as a potential conflict of interest.

Received: 25 October 2011; paperpending published: 13 November 2011; accepted: 15 December 2011; published online: 03 January 2012.

Citation: Gilbert RJ, Mitchell MR, Simon NW, Bañuelos C, Setlow B and Bizon JL (2012) Risk, reward, and decisionmaking in a rodent model of cognitive aging. Front. Neurosci. 5:144. doi: 10.3389/fnins.2011.00144

This article was submitted to Frontiers in Decision Neuroscience, a specialty of Frontiers in Neuroscience.

Copyright (๑) 2012 Gilbert, Mitchell, Simon, Bañuelos, Setlow and Bizon. This is an open-access article distributed under the terms of the Creative Commons Attribution Non Commercial License, which permits non-commercial use, distribution, and reproduction in other forums, provided the original authors and source are credited. 